\title{
Deep Resection Margin
}

National Cancer Institute

\section{Source}

National Cancer Institute. Deep Resection Margin. NCI Thesaurus. Code C138062.

The surgical margin resulting from resection of a tumor with respect to the deep border of the specimen, as it was oriented prior to excision. 